\title{
Treatment outcome of severe malaria in adults with emphasis on neurological manifestations at Gondar University Hospital, north west Ethiopia
}

\author{
Getahun Mengistu ${ }^{1}$, Ermias Diro ${ }^{2}$
}

\begin{abstract}
Background: Malaria is a major health threat in Ethiopia. Unstable malaria transmissions cause severe clinical manifestations and result in high case fatality.

Objective: The aim of this study was to asses the clinical features and treatment outcome of severe malaria cases with special emphasis on the neurologic manifestations.

Method: A retrospective medical records analysis was done at Gondar University hospital, which is located in Gondar town.

Results: Severe malaria accounted for $4.4 \%$ of the admissions and for $7.3 \%$ of the deaths occurring in the medical wards of the hospital. Neurological manifestations were observed in $61.5 \%$ of cases. The crude case fatality rate was $28.4 \%$ with $87.1 \%$ of the deaths having neurologic manifestations. Cerebral malaria accounted for $58.1 \%$ of the deaths and it was the most significant independent variable predicting poor outcome. Other poor prognostic indicators were late presentation to the hospital, acidotic breathing, pulmonary edema, shock or/hypotension, hyperparasitemia, rural residence and age 40 years and above in adults.

Conclusion and recommendations: Neurological manifestations were the major determinant of morbidity and mortality in severe malaria cases in adults. This study indicates that more emphasis needs to be given to prevention and early management of malaria. Priority should also be given to patients with neurologic manifestations and they should be cared for in intensive care units. [Ethiop.J.Health Dev. 2006;20(2):106-111]
\end{abstract}

\section{Introduction}

Malaria remains a heavy burden on tropical communities. According to WHO estimates the global incidence of malaria in 2004 was between 350 million to 500 million cases $(1,2)$. Almost all of the deaths were attributable to Plasmodium falciparum and is the only species that appears to directly affect the central nervous system causing neurologic deficits, cognitive sequel and epilepsy $(3,4,5,6)$.

About $90 \%$ of the world's malaria deaths are estimated to occur in sub-Saharan Africa where the majority of the infections are caused by the most dangerous species, $P$. falciparum, (2). In Africa more than twelve billion dollars is lost every year in GDP due to malaria (7). The economic burden of malaria to the countries, the families and the individuals is immense. Malaria strikes during the planting and harvesting seasons $(2,8)$.

In Ethiopia many millions people live in areas of seasonal and less predictable malaria transmission commonly known as fringe areas. Populations in these areas generally have lower levels of immunity and all age groups are vulnerable to highly seasonal transmission and epidemics $(2,8)$. Reduction in transmission intensity reduces immunologic stimulation, which may have longterm effects of shifting the peak in the age incidence profiles to older ages and severe diseases with epidemic outbreaks (8-11). Poor populations are at greatest risk; of death and those patients receive the worst care $(9,12)$.
According to the data obtained from health institutions operating in north Gondar, $P$. falciparum malaria accounted for $64.4 \%$ of the cases with $35.6 \%$ caused by $P$. vivax (13). $P$. falciparum malaria is among the leading causes of hospitalization and hospital deaths (14) and accounts for almost all cases of severe and complicated malaria.

The clinical picture of severe malaria in African adults is not well documented but, in most settings, impaired consciousness is a major presentation. Prostration is the milder end of the spectrum of impairments of neurologic function ranging from lethargy to deep coma (15).

The central nervous system involvement in $P$. falciparum malaria is the most common complication and cause of death responsible for $10 \%$ of the admissions and $80 \%$ of deaths (16). Cerebral malaria compounded with hypoglycemia, multiple convulsions more than twice in 24 hours and impaired state of consciousness with behavioral change; confusion and stupor are the neurological manifestations of severe and complicated malaria (3, 16, 17, 18). Cerebral malaria a rapidly evolving neuroligic disease and the hallmark of Plasmodium falciparum infection carries a fatality rate of more than $20 \%$ even in urban hospital settings where aggressive medical care is available $(3,19)$. Hypoglycemia, convulsion, respiratory distress, circulatory collapse, metabolic acidosis and renal failure

\footnotetext{
${ }^{1}$ Department of Neurology, Addis Ababa University Faculty of Medicine, E-mail getahaau@yahoo.com;

${ }^{2}$ Departmet of Internal Medicine, Gondar University, Tel 0911670953, P.O. Box 14428, Addis Ababa,

E-mail ermi_diro@yahoo.com
} 
may aggravate or contribute to impaired consciousness in severe malaria $(3,15-18)$.

In adults treated in1991 at Gondar college hospital, neurological manifestations were observed in about $80 \%$ of the severe malaria cases and the case fatality rate of cerebral malaria was 77\% (23/30)(20).

Data on the neurological and other severe manifestations of malaria in Ethiopia are scarce especially for adults. Hence the objective of this study was to asses the outcome of neurological manifestations of severe and complicated malaria among adults admitted to Gondar University hospital.

\section{Subjects and Methods}

Retrospective record analysis of severe malaria patients at Gondar University Hospital was made. Gondar University hospital is located in Gondar town $750 \mathrm{~km}$ from Addis Ababa Northwest of Ethiopia. It is both a teaching and referral hospital with 350 beds, 70 beds are located in the medical wards.

The records and charts of patients with severe and complicated malaria over six years period from September 1, 1998 to August 31, 2004 were retrieved from the discharge and death summary records and the ward registry books. Data were collected using formats prepared for this purpose. The format included demographic parameters, the WHO severity criteria (clinical and laboratory) as well as outcome and hospital stays.

All admitted patients with positive asexual form of $P$. falciparum malaria and at least one of the following were included $(1,3,19)$ :

1. Cerebral malaria: unrousable coma (Glasgow coma scale $\leq 9$ ) that lasted more than 30 minutes after convulsion in falciparum malaria, not attributable to any other cause.

2. Multiple convulsions: more than two generalized seizures in 24 hours-occurring not due to causes other than malaria.

3. Arousable degree of impaired consciousness with behavioral change, confusion or drowsiness (Glasgow coma scale $>9$ ).

4. Pulmonary edema: acute respiratory distress syndrome.

5. Acidosis: labored deep breathing.

6. Normocytic normochromic anemia: Hematocrit < $20 \%$.

7. Renal failure: serum creatinine $>3 \mathrm{mg} \%$.

8. Hypotension: systolic blood pressure $<80 \mathrm{mmHg}$.

9. Bleeding: significant bleeding and hemorrhage from the gums, nose, and gastro intestinal tract or evidence of disseminated coagulopathy.

10. Hemoglobinuria: macroscopic black, brown, or red urine.
11. Prostration/extreme weakness: inability to walk or sit up without assistant. This is the milder and benign end of the neurological manifestations spectrum, potentially dangerous unless treated urgently.

12. Hyperparasitemia: parasite load $>5 \%$.

13. Jaundice: serum bilirubin level $>3.0 \mathrm{mg} \%$.

Analysis of arterial blood gas and lactate level were not done, as there were no facilities. There was no intensive care unit. All patients were admitted and cared in medical wards.

For data entry and analysis, EPI-Info 2002 and SPSS 10.0 statistical packages were used. Comparison of severity manifestation with the outcome was done. Logistic regression model was utilized to identify predictors and $\mathrm{p}<0.05$, odds ratio (OR) (95\% confidence interval) and T-test used for significance.

\section{Results}

There were a total of 9322 admissions and 1584 deaths in the medical wards, at Gondar University hospital over the six-year period. Of these, $408(4.4 \%)$ of the cases and $116(7.3 \%)$ of the deaths were due to severe and complicated malaria. Females were 223(54.7\%) and 185(45.3\%) were males. The mean age was 32.2, ranging between 14 and 80 years (Table 1). Mean age of those who died was significantly higher than those who survived, $35.3 \pm 14.1$ vs $31.0 \pm 12.5(\mathrm{~T}=3.0247, \mathrm{p}=0.002)$. Out of the total 408 cases, 238 (58.3\%) were from the rural area where as the rest $170(41.7 \%)$ were urban dwellers. The mean duration of illness before admission was 6.6+4.6 days. The mean parasitic load was 8.5 $\pm 11.9 \%$. This was significantly higher in those who died than in those who survived (13.5 $\pm 15.6 \%$ vs $6.6 \pm 9.5 \%)$ $\mathrm{T}=4.9, \mathrm{p}=0.0000$. The mean duration of parasitic clearance was $2.6 \pm 1.6$ days with the median of 2 days.

$\begin{aligned} & \text { Table 1: Age and sex distribution of severe and } \\
& \text { complicated malaria patients in Gondar University } \\
& \text { Hospital, 1998-2004 }\end{aligned}$
\begin{tabular}{llll} 
Age group & Female (\%) & Male (\%) & Total (\%) \\
\hline $10-19$ & $31(13.9)$ & $18(9.7)$ & $49(12.0)$ \\
$20-29$ & $93(41.7)$ & $56(30.3)$ & $149(36.5)$ \\
$30-39$ & $70(31.4)$ & $41(22.2)$ & $111(27.2)$ \\
$40-49$ & $12(5.4)$ & $31(16.8)$ & $43(10.5)$ \\
$50-59$ & $9(4.0)$ & $21(11.4)$ & $30(7.4)$ \\
$\geq 60$ & $8(3.6)$ & $18(9.7)$ & $26(6.4)$ \\
\hline Total & $\mathbf{2 2 3 ( 5 4 . 7 )}$ & $\mathbf{1 8 5 ( 4 5 . 3 )}$ & $\mathbf{4 0 8 ( 1 0 0 . 0 )}$ \\
\hline
\end{tabular}

Out of the total 408 cases of severe and complicated malaria, 121 (29.7\%) had single WHO defined severity indicator while $287(70.3 \%)$ had two or more overlapping indicators. Of all the cases, 251(61.5\%) had the overlapping neurological manifestations, $117(28.7 \%)$ had cerebral malaria, 51(12.5\%) repeated convulsions and 129 (31.6\%) manifested in change of behavior, confusion or drowsiness. Prostration, the milder form in the 
spectrum of neurologic manifestations, accounted for 129 (31.6\%) (Table 2).

Table 2: Clinical and laboratory manifestations of severe and complicated malaria in patients at Gondar University Hospital, 1998-2004, N=408

\begin{tabular}{lll}
\hline Manifestation & Number & Percent \\
\hline $\begin{array}{l}\text { Behavioral change, confusion or } \\
\text { drowsiness }\end{array}$ & 129 & 31.6 \\
Cerebral malaria & 117 & 28.7 \\
Multiple convulsions & 51 & 12.5 \\
Sub total (all neurological & $\underline{251}$ & $\underline{61.5}$ \\
manifestations & & \\
Hyperpasitemia & 130 & 31.9 \\
Prostration & 129 & 31.6 \\
Normocytic normochromic anemia & 112 & 27.5 \\
Jaundice & 87 & 21.3 \\
Hypotension & 52 & 12.7 \\
Hemoglobinuria & 33 & 8.1 \\
Pulmonary edema & 30 & 7.4 \\
Acidotic breathing & 21 & 5.1 \\
Renal failure & 15 & 3.7 \\
Hypoglycemia & 13 & 3.2 \\
Bleeding tendency & 9 & 2.2 \\
\hline Total & $\mathbf{4 0 8}$ & \\
\hline
\end{tabular}

NB: all the neurological manifestations observed add up to more than 251 because more than one may occur in the same patient.
The over all case fatality rate of severe malaria was $28.4 \%(116 / 408)$. The gender specific fatality rates were $63 / 223(28.3 \%)$ in females and 53/185(28.6\%) in males.

The case fatality rate of all patients that had neurological manifestations was $101(40.2 \%)$. The case fatality rate of cerebral malaria was 68/117 (58.1\%) those with convulsions was 19(37.3\%) and the change of behavior 32(24.5\%) (Table 3).

Of the total 116 deaths, 101(87.1\%) displayed at least one of the neurological manifestations. About 68(58.6\%) of the deaths had cerebral malaria with or without multiple convulsions. Cerebral malaria when accompanied by convulsions accounted for as high as $55.2 \%$ of the deaths (Table 4 ). Cerebral malaria, acidotic breathing pulmonary edema jaundice, hypotension, hyperparasitemia, rural residence and age 40 years or above were bad prognostic indicators (Table 5).

Table 3: Case fatality and, Clinical and laboratory manifestations of severe and complicated malaria in Gondar University Hospital inpatients, 1998- 2004, N=408

\begin{tabular}{llll}
\hline Manifestation & Cases & Deaths & Case fatality rate (\%) \\
\hline Change of behavior & & 32 & 24.8 \\
Cerebral malaria & 129 & 68 & 58.1 \\
Multiple convulsions & 117 & 19 & 37.3 \\
Sub total (all neurological manifestations) & 51 & $\mathbf{2 5 1}$ & $\mathbf{4 0 . 2}$ \\
Prostration & 129 & 101 & 40.0 \\
Hyperpasitemia $>5 \%$ & 130 & 52 & 22.3 \\
Anemia & 112 & 25 & 40.2 \\
Jaundice & 87 & 35 & 51.9 \\
Hypotension & 52 & 27 & 12.1 \\
Hemoglobinuria & 33 & 4 & 80.0 \\
Pulmonary edema & 30 & 24 & 85.7 \\
Acidotic breathing & 21 & 18 & 40.0 \\
Renal failure & 15 & 6 & 46.2 \\
Hypoglycemia & 13 & 6 & 55.6 \\
Bleeding tendency & 9 & 5 & 34.5 \\
Rural patients & 238 & 82 & 38.4 \\
Age $\geq 40$ years & 99 & 38 & 45.9 \\
Hospital stay $<7$ days & 220 & 101 & $\mathbf{2 8 . 4}$ \\
\hline Total & $\mathbf{4 0 8}$ & $\mathbf{1 1 6}$ & \\
\hline
\end{tabular}

NB: all neurological manifestations added up to more than 251, because more than one manifestation occurred in the same patient.

\section{Discussion}

In the six-year admissions at Gondar University Hospital, there were 408 cases of severe and complicated adult malaria, out of those 116 had died. This accounted for about $4.4 \%$ of the admissions and $7.3 \%$ of the deaths in the medical wards of Gondar University Hospital. It was a burden for the hospital in general and the medical ward in particular where there is no intensive care unit.
The mean age was 32 years but those who died had a significantly higher age than the survivors. With one or more seasonal or unstable malaria transmission patterns, the age distribution of severe malaria shifts upward, severe malaria is seen in older children and adults as well, and cerebral malaria becomes the most prominent manifestation (21). In this study about $86 \%$ and $76 \%$ of the cases were below 50 and 40 years of age respectively. 
Table 4: Case fatality and neurological manifestations in severe and complicated malaria at Gondar University Hospital, 1998-2004

\begin{tabular}{lccc}
\hline Neurological manifestations & No. of cases & No. of deaths & CFR (\%) \\
\hline Change of behavior, confusion or drowsiness & 116 & 30 & 25.9 \\
Cerebral malaria & 84 & 52 & 61.9 \\
Multiple convulsions $>2$ / 24 hr. & 5 & 1 & 20.0 \\
Change of behavior, plus multiple convulsions. & 13 & 2 & 15.4 \\
Cerebral malaria plus multiple convulsions >2 / 24 hours & 33 & 16 & 48.5 \\
\hline Total & 251 & 101 & 40.2 \\
\hline CFR = case fatality rate & &
\end{tabular}

Table 5: Factors predicting mortality from severe and complicated malaria in Gondar University Hospital 19982004

\begin{tabular}{lllll}
\hline Characteristics & & Number & Death, N (\%) & Adjusted OR( 95\% Cl) \\
\hline Cerebral malaria & no & 291 & $48(16.5)$ & 1 \\
& yes & 117 & $68(58.1)$ & $6.4(3.7-11-4)$ \\
Hyperpasitemia & $<5 \%$ & 278 & $64(23.0)$ & 1 \\
& $\geq 5 \%$ & 130 & $52(40.0)$ & $2.1(1.2-3.6)$ \\
Acidotic breathing & no & 387 & $98(25.3)$ & 1 \\
& yes & 21 & $18(85.7)$ & $13.4(3.2-56.3)$ \\
Pulmonary edema/ARDS* & no & 378 & $93(24.6)$ & 1 \\
& yes & 30 & $24(80.0)$ & $5.2(1.8-15.3)$ \\
Hypotension & no & 356 & $89(25.0)$ & 1 \\
\multirow{2}{*}{ Jaundice } & yes & 52 & $27(51.9)$ & $2.7(1.3-5.6)$ \\
\multirow{2}{*}{ Age in years } & no & 321 & $81(25.2)$ & 1 \\
& yes & 87 & $35(40.2)$ & $2.7(1.5-5.1)$ \\
Address & $<40$ & 309 & $78(25.2)$ & 1 \\
& $\geq 40$ & 99 & $38(38.4)$ & $1.8(1.0-3.2)$ \\
& Urban & 170 & $34(20.0)$ & 1 \\
\hline
\end{tabular}

${ }^{*}$ ARDS=Acute Respiratory Distress Syndrome

This has its own impact on the economic growth and population dynamic as the productive and reproductive segment is most affected.

In this study, close to $42 \%$ of the cases were urban dwellers, which is much higher than that reported in various population studies and estimates in Africa. It is known that between $6 \%$ and $28 \%$ of the malaria burden occurring in cities, which comprises less than $2 \%$ of the African surface area (9). However, this may not reflect the true malaria burden as it was retrospective and hospital based severe case only. It may also be affected by limitations in accessing health services.

Taking all the overlapping features together, neurological manifestations were the most common presentation of severe and complicated malaria accounting for almost $62 \%$. These in decreasing order were behavioral change, cerebral malaria and convulsions. In this study cerebral malaria occurred in about $29 \%$ of the cases and is similar to that of the Saudi Arabia region bordering the red sea which is $26 \%$ (22) but lower than the Veitnamese figure of $52 \%$ (23). Multiple convulsions, which occurred in $13 \%$ of the cases in this study, are similar to $11.3 \%$ of the cases in Vietnam. Among all cases of cerebral malaria in this study $28 \%$ had multiple convulsions, which is higher than the prospective Indian study in adults that occurred in $21 \%$ (24). Convulsions in malaria are often recurrent and $84 \%$ of the seizures are complex, most often with a focal nature (5).

Hyperparasitemia or parasite load more than 5\% comprised about $32 \%$ of the cases. Normochromic normocytic anemia was found in $28 \%$, which is higher than the $17 \%$ in Saudi Arabia but similar number of cases of jaundice (21\% vs 24\%) were observed (22). Parasitemia can be higher than $50 \%$ in P.falciparum and in nonimmune individuals greater than $5 \%$ is associated with severe disease (25).

The overall case fatality rate was $28.4 \%$ with no gender difference. This is lower than the study undertaken in 1991 in Gondar, where it was 51\% (20) but it was 10 times higher than the study undertaken in Saudi Arabia (22) and twice higher than the 1996 study in Vietnamese adults (23). The case fatality rate and proportionate mortality rate for hospitalized cases of all types malaria patients (severe, non-severe) were $20 \%$ and 56\% 
respectively according to a study done in 2005 in Southern Oromia (26). Hospitals of secondary or tertiary referral centers often experience high mortality rates as they see of more severe patients (21). Mortality is also dependent on the availability of an intensive care unit (5); lack of this facility in Gondar hospital might have contributed to the observed high mortality in this study.

This study showed that $87 \%$ of the deaths had one or more of the neurological manifestations. Cerebral malaria (with or without convulsion) accounted for about $59 \%$ of the overall deaths. Many adults with neurological manifestations of malaria in general and cerebral malaria in particular die from pulmonary edema, renal failure, acute respiratory distress or arrest (27). The central nervous system involvement is the most common complication and cause of death in severe $P$. falciparum infection, accounting for $10 \%-20 \%$ of all admissions and $80 \%$ of the deaths from falciparum malaria $(21,28)$ cerebral malaria being the most common and life threatening neurological complication (18).

The case fatality of cerebral malaria was close to $58 \%$ followed by that of all neurological manifestations $(40 \%$ fatality). This was much higher than earlier findings in Ethiopia and elsewhere which ranged from $10-20 \%$ in adults $(1,3,5,28,29)$.

The highest case specific fatality rates $86 \%$ and $80 \%$ were found in acidotic breathing and pulmonary edema, respectively. Both were found to be independent predictors of death. Acidotic breathing which is hyperventilation with deep breathing may develop in severely ill who are shocked, hypoglycemic, hyperparasitemic or in renal failure due to lactic acidosis in both blood and CSF $(3,4)$. Pulmonary edema/ARDS can develop at any time in falciparum malaria, even after several days of antimalarial treatment. It is a very grave complication with a mortality rate more than $80-85 \%$ (4, 30).

Hypotension /shock (often called algid malaria) occurred in $13 \%$ of patients with a mortality rate of $52 \%$ and it was another independent factor of mortality. The mortality rate remains high in which a proportion of cases might have septicemia (21).

Jaundice was a manifestation in $21 \%$ with a case specific fatality of $40 \%$, independently predicting death. Death due to hepatic coma and serum bilirubin levels up to $25 \mathrm{mg} / \mathrm{dl}$ have been observed more common in adults than children. Jaundice and hepatic dysfunction are important determinants of mortality in cerebra malaria $(21,30)$.

Hyperparasitemia of $5 \%$ or more was found in 32\% with a case specific fatality of $40 \%$, again independently predicting death. More than $10 \%$ parasite load indicates potentially dangerous infection irrespective of other factors in unstable malaria area (31).

The majority of the deaths occurred in the first few days of hospitalization. Most deaths of severe malaria especially cerebral malaria occur within 24- 48 hours of admission (5). These factors are related to severity of illness and occurrence of irreversible complications.

In conclusion, this study showed that severe malaria is a major burden of Gondar hospital. Many of the severe signs described by WHO were seen in the patients studied, of which the neurological manifestations and central nervous system involvement were predominant. Neurological complications (particularly the cerebral malaria), acidotic breathing, and pulmonary edema, were the major poor prognostic indicators in adults. It is thus recommended that identification of severity and its type be used in prioritization of care, with emphasis on neurological complications. Access to health services, early and appropriate treatment before complications are crucial. It is needless to say opening an intensive care unit is of paramount importance in the hospital.

\section{Acknowledgements}

We would like to thank the Gondar university research and publication office for supporting the study. Our gratitude is extended to Dr. Alemayehu Worku of Department of community health, Addis Ababa University for his help in statistical analysis and revising the manuscript. We thank Dr. Janet T. Jones from Glasgow University, Scotland for revising and editing the manuscript.

\section{References}

1. White, NJ, Berman, J.G: Malaria and babesiosis: diseases caused by red blood parasites, In D.L. Kasper, E. Braunwald, A.S.Fauci, S.L.Hauser.D.L. Longo and J.L.Jemson (eds). Harrison's principles of internal medicine 16th edn.2005;1:1218-1233.

2. World Health Organization. Malaria control today; current WHO recommendations, working document; Roll Back Malaria Department; WHO, Geneva; 2005 pages 1-75.

3. World Health Organization. Severe and complicated malaria third edition; Trans R Soc Trop Med Hyg 2000;94(suppl-1):1-90.

4. World Health Organization. Management of severe malaria; a practical handbook, second edition, WHO, Geneva, 2000.

5. Newton CR, Hien TT, White N; Cerebral malaria J Neurol Neurosurg Psychiatry 2000;69:433-441.

6. Ala-Odera VM, Snow RW, and Newton CR; the burden of Neurocognitive impairment associated with Plasmodium Malaria in sub-Saharan Africa; Am J Trop Med Hyg 2004;71(suppl 2):64-70.

7. World Health Organization. Malaria in Africa; Roll Back Malaria Inf0sheet; 2005;http//www.rbm.int/.

Ethiop.J.Health Dev 2006;20(2) 
8. Kiszewski, AE and Teklehaimanot A; Review of the clinical and epidemiologic burden of epidemic malaria; Am J Trop Med Hyg; 2004;71(suppl 2):128-135.

9. Breman JG, Alilio MS and Mills A Conquering the intolerable burden of malaria: what is new, what is needed: a summary Am J Trop Med Hyg; 2004;71(suppl 2):1-15.

10. Smith T, Killeen G, Lengeler C, and Tanner M; Relationship between outcome of Plasmodium falciparum infection and the intensity of transmission in Africa; Am J Trop Med Hyg; 2004;71(suppl 2): 80-86.

11. Worrall E, Rietveld A, and Delacollette C; The burden of malaria epidemics and cost effectiveness of interventions in epidemic situations in Africa; Am J Trop Med Hyg 2004;71(suppl 2):136-140.

12. Barat LM, Palmer N, Basu S, Worrall E, Hanson K, and Mills A; Do malaria control interventions reach the poor? A view through equity lens; Am J Trop Med Hyg 2004;71(suppl 2):174-178.

13. Mitiku K, Mengistu G, Gelaw B; The reliability of blood film examination for malaria at the peripheral health units; Ethiop J Health Dev; 2003;17(3):197204.

14. Ministry of Health; Malaria diagnosis and treatment guidelines for health workers in Ethiopia $\mathrm{MOH}$; July 1999:1-54.

15. Marsh K, and Makani J, Malaria; in E. Parry, R. Godfrey, D. Mabey and G. Gill (Edn); Principles of medicine in Africa; third Edn, Cambridge University press; 2004: 284-304.

16. Central nervous system involvement in falciparum malaria; Dr.B.S.Kakkilaya malaria Website 20022004.

17. Sotelo ,J and Willms, K Parasitic diseases; In A.K. Asbury, G.M. McKhann, W.I. McDonald, P.J. Goadsby (edn); Diseases of the nervous system 3rd edn., 2002;2:1745-1753.

18. Garg RK, Karak B, Misra S, Neurological manifestations of malaria: an update. Neurol India (serial on line) 1999;47:85-91.

19. Warell DA, Molyneux ME, Beales PF, severe and complicated malaria second edition; Trans $\mathrm{R}$ Soc Trop Med Hyg 1990;84 (suppl-2):1-65.
20. Endeshaw Y, Seyoum A, Amanuel B Clinical and laboratory features of severe and complicated malaria , the experience from Gondar; Ethiop Med J, 1991;29(1):21-26.

21. White, NJ; Malaria; In G.C Cook and A. Zumla (edn); Manson's Tropical diseases $21^{\text {st }}$ edition; 2003:1205-1295.

22. Banzal S, Ayoola EA, El Sammani EE, Rahim SI, Subramaniam P, Gadour M O E, and Jain A K; The clinical pattern and complications of severe malaria in the Gizan region of Saudi Arabia, Annals of Saudi Medicine 1999;18(4):378-380.

23. Hein TT, Day NPJ, Phu NH etal, A controlled trial of artemether or quinine in Vietnamese adults with severe falciparum malaria; $N$ Eng $\mathrm{J}$ Med 1996:335:76-83.

24. Kochar DK, Shubhakaram, Kumawat BL, Kochar SK, Halwai M, Makkar RK, Joshi A, Thanvi I, Cerebral malaria in Indian adults: a prospective study of 441 patients from Bikaner, north-west India, Assoc Physicians India; 2002;50:234-241.

25. Trampuz A, Jereb M, Muzlovic I, and Prabhu RM, Clinical review: severe malaria, Critical care, 2003;7:315-323.

26. Deressa, W, Olana, D and Chibsa, S. Community participation in Malaria epidemic control I highland areas of Southern Oromia, Ethiopia; Ethiop J Health Dev 2005:19(1):3-30.

27. Idra R, Jenkins N, Newton RJC, Pathogenesis, clinical features and neurological out come of cerebral malaria. Lancet Neurol 2005;4:827-840.

28. Kakkilaya BS. Central Nervous System Involvement in falciparum malaria; 2002; malaria web site.

29. Mengistu M, Maru M, Ahmed Z; Malaria in Gondar, Ethiopia, 1975-1978; a review of 435cases with special emphasis on cerebral malaria; Ethiop Med J 1979;17(3):57-62.

30. Shukia MM, Singh N, Singh MP et al. Cerebral malaria in Jabalpur. Ind J Malarialogy, 1995; 32:705.

31. Patel DN, Pradeep P, Surti MM, Agarwal SB; Clinical manifestations of Complicated malaria; An Overview, Journal, Indian Academy of Clinical Medicine; 2003;4(4):323-31. 